\title{
Solitary Electromagnetic Pulses Detected with Super-Alfvénic Flows in Earth's Geomagnetic Tail
}

\author{
G. K. Parks, ${ }^{*}$ E. Lee, N. Lin, F. Mozer, and M. Wilber \\ Space Sciences Laboratory, University of California, Berkeley, California, USA \\ I. Dandouras and H. Rème \\ Centre d'Etude Spatiale des Rayonnement, Paul Sabatier University, Toulouse, France \\ E. Lucek \\ Blackett Laboratory, Imperial College, London, United Kindom \\ A. Fazakerley \\ Mullard Space Sciences Laboratory, University College London, Dorking, United Kindom \\ M. Goldstein \\ NASA Goddard Space Flight Center, Greenbelt, Maryland, USA \\ C. Gurgiolo \\ Bitterroot Basic Research Inc., 837 Westside Road, Hamilton, Montana, USA \\ P. Canu and N. Cornilleau-Wehrlin \\ CETP, Velizy, France \\ P. Décréau \\ LPCE and University of Orleans, Orleans, France \\ (Received 29 March 2007; published 27 June 2007)
}

\begin{abstract}
Solitary nonlinear $(\delta B / B \gg 1)$ electromagnetic pulses have been detected in Earth's geomagnetic tail accompanying plasmas flowing at super-Alfvénic speeds. The pulses in the current sheet had durations of $\sim 5 \mathrm{~s}$, were left-hand circularly polarized, and had phase speeds of approximately the Alfvén speed in the plasma frame. These pulses were associated with a field-aligned current $J_{\|}$and observed in low density $\left(\sim 0.3 \mathrm{~cm}^{-3}\right)$, high temperature $\left(T_{e} \sim T_{i} \sim 3 \times 10^{7} \mathrm{~K}\right)$, and $\beta \sim 10$ plasma that included electron and ion beams streaming along $\mathbf{B}$. The wave activity was enhanced from below the ion cyclotron frequency to electron cyclotron and upper hybrid frequencies. The detailed properties suggest the pulses are nonlinearly steepened ion cyclotron or Alfvén waves.
\end{abstract}

DOI: 10.1103/PhysRevLett.98.265001

PACS numbers: 94.05.Fg, 94.20.wc, 94.20.wf, 94.30.ct

We present first observations of solitary electromagnetic (EM) pulses detected in the Earth's geomagnetic tail during intervals of super-Alfvénic flows, where the perpendicular flow $V_{\perp}$ exceeds the Alfvén speed $V_{A}$. Most flow speeds are sub-Alfvénic and can be explained by reconnection theories and models [1]. However, plasmas occasionally flow faster than $V_{A}$ [2]. The significance of $V_{\perp}>V_{A}$ flows and the possible nonlinear effects that might accompany them have not been discussed. Solitary waves are signatures of nonlinear interactions, and they can shed light on the elusive current sheet dynamics that drive the global aurora when Earth becomes an intense radio emitter and electrons are accelerated to hundreds of $\mathrm{keV}$ and ions to several $\mathrm{MeV}[3,4]$.

Solitary EM waves have been observed in comets and also in Earth's ionosphere [5,6], and Debye scale electrostatic solitary waves are ubiquitous in the solar wind, bow shock, plasma sheet boundary layer (PSBL), and the auroral ionosphere [7-9]. Unipolar and bipolar wave packets have been detected in the PSBL, and magnetosonic solitons at the magnetopause $[10,11]$. However, there have been no reports of solitary pulses accompanying flows in which $V_{\perp}>V_{A}$ in the Earth's geomagnetic tail.

A random sampling of cluster data of fast flowing plasmas indicates that $V_{\perp}>V_{A}$ flows occur when the geomagnetic tail is undergoing large fluctuations (but $V_{\perp}>V_{A}$ speeds are not always observed when and where large fluctuations occur). High-time resolution $\mathbf{E}$ and $\mathbf{B}$ fields during a number of events indicate that the EM pulses occur concurrently with $V_{\perp}>V_{A}$ flows in the current sheet (CS) while electrons and ions are streaming along $\mathbf{B}$ and wave activity is enhanced.

The data in Fig. 1 are spin-averaged (4 s) from Cluster 1 (SC1) located at a tail distance of $\sim 19 R_{E}\left(R_{E}=\right.$ Earth's radius). The ion bulk parameters are obtained by integrating the 3D distributions over the velocity space [12]. Measurements here were made in a thin CS $(\sim 400 \mathrm{~km})$ during an aurora [13]. SC1, initially in the plasma sheet 


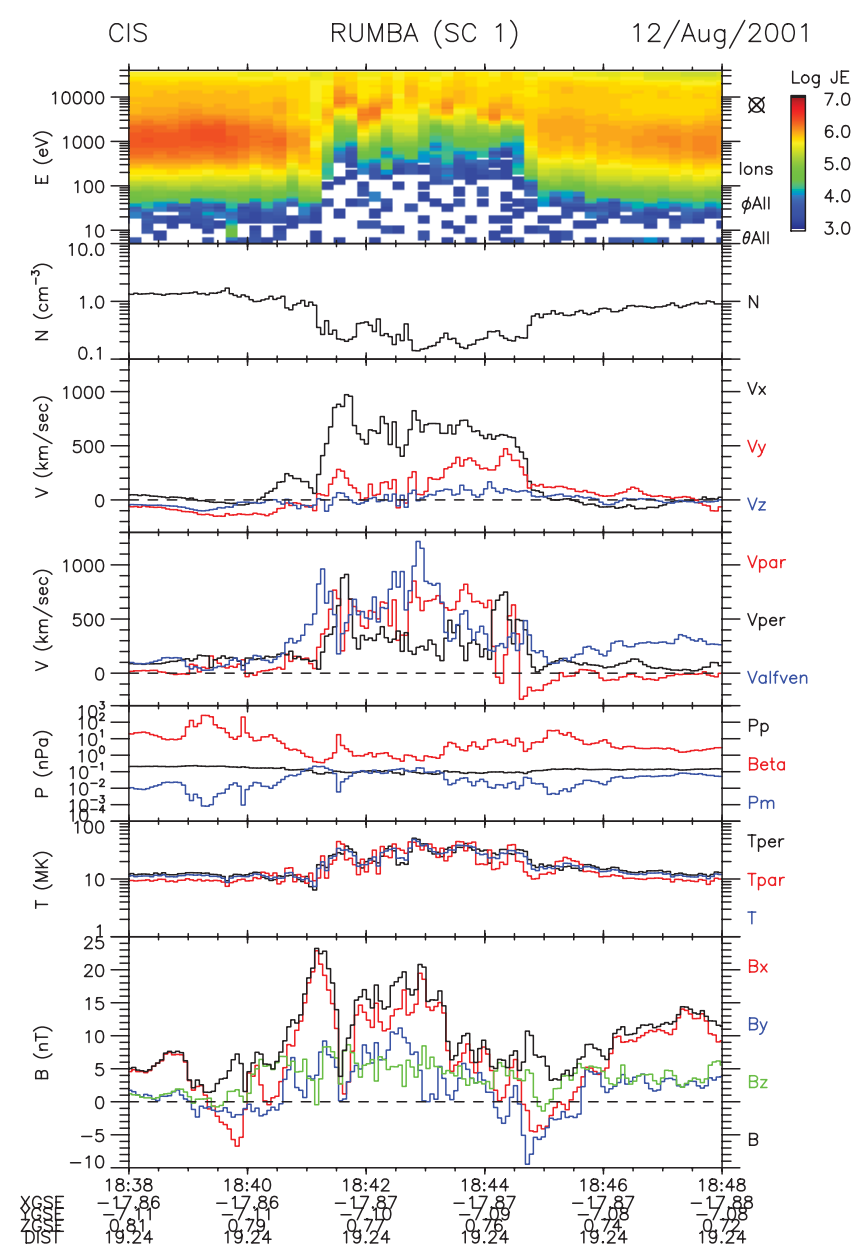

FIG. 1 (color). Ions measured from a few $\mathrm{eV} / q$ to $45 \mathrm{keV} / q$. Top to bottom: (1) Energy flux spectrogram, (2) density, (3) bulk velocities, (4) bulk speeds parallel and perpendicular to $\mathbf{B}$ and $V_{A}$, (5) plasma and magnetic pressure, and beta of plasma, (6) temperature, $T_{\|}, T_{\perp}$, total $T$, and (7) magnetic field components and intensity.

(PS), entered a rarefied hot plasma region (HPR) at $\sim 1841$ UT, remaining in it until $\sim 1845$ UT before returning to the PS. The HPR is characterized by low fluxes of $<2 \mathrm{keV}$ ions, which accounts for the high temperatures $T$ and anticorrelated densities $n$. Moreover, the high bulk velocities $\left(1000 \mathrm{~km} \mathrm{~s}^{-1}\right)$ are mostly along $V_{x}$ (sunward). The velocities parallel $\left(V_{\|}\right)$to $\mathbf{B}$ and $V_{\perp}$ at times exceeded $V_{A} . V_{\|}>V_{A}$ has been seen previously [14], and here we focus only on $V_{\perp}>V_{A}$. At 1841:30 UT, plasma $\beta$ increased to $\sim 30$, and $V_{\perp}$ reached $\sim 800 \mathrm{~km} \mathrm{~s}^{-1}$ attaining an Alfvénic Mach number of $\sim 4.0 . V_{\perp}$ also exceeded the fast mode speed $\sim 557 \mathrm{~km} \mathrm{~s}^{-1}$. Typical plasma parameters in the HPR (PS) were $n_{i} \sim 0.5(1.5) \mathrm{cm}^{-3}, T_{i} \sim 3 \times$ $10^{7}\left(1.2 \times 10^{7}\right) \mathrm{K}, T_{e} \sim 5 \times 10^{6}\left(2 \times 10^{6}\right) \mathrm{K}, B \sim 15(8) \mathrm{nT}$, $\beta=10(30), \quad V_{A} \sim 200(100) \mathrm{km} \mathrm{s}^{-1}, \quad$ and $\quad C_{s} \sim$ $210(150) \mathrm{km} \mathrm{s}^{-1}$.

The $\mathbf{B}$ measurements [15] indicate that activity started before entry into HPR, $\sim 1838$ UT. Large excursions of $B_{x}$ allowed SC1 to cross the CS (determined by a change of sign in $\left.B_{x}\right)$ as well as to sample the PSBL $\left(B_{x} \sim 24 \mathrm{nT}\right)$. $X, Y, Z$ are geocentric solar ecliptic (GSE) coordinates. The $B$ field inside the HPR was usually intense and turbulent and included sharp pulses, several of which were associated with $V_{\perp}>V_{A}$. The pulse accompanying $V_{\perp}>$ $V_{A}$ at 1841:30 UT detected by SC2 is shown in Fig. 2. At the same time, SC3 in the PSBL detected a broader pulse. $\mathrm{SC} 1$ detected rapid fluctuations, and SC4 did not see any pulses (not shown).

The time profiles of $\delta B_{x}$ and $\delta B_{z}, \delta E_{y}$ and $\delta E_{z}$ are bipolar, with $\delta B_{y}$ unipolar. Minimum (maximum) variance analysis applied to $\delta \mathbf{B}(\delta \mathbf{E})$ yielded a normal direction $\mathbf{k}=(0.781,-0.336,0.526)$ that was $\sim 45^{\circ}$ from $\mathbf{B}$. In the plane normal to $\mathbf{k}, \delta \mathbf{E}$ and $\delta \mathbf{B}$ were $\sim 90^{\circ}$ to each other, and the fields rotated together, a signature of an EM wave. The fields rotated circularly by $\sim 360^{\circ}$ in the left-hand sense within the pulse duration of 5-7 s, which coincidentally was the Larmor period of protons in the average pulse field $\sim 12 \mathrm{nT}$.

The phase speed along $\mathbf{k}, V_{\mathrm{ph}}=\mathbf{k} \cdot \delta \mathbf{E} \times \delta \mathbf{B} / \delta B^{2}$, had the largest component in the sunward direction $\left(\sim 1000 \mathrm{~km} \mathrm{~s}^{-1}\right)$. The Doppler shifted speed in the plasma

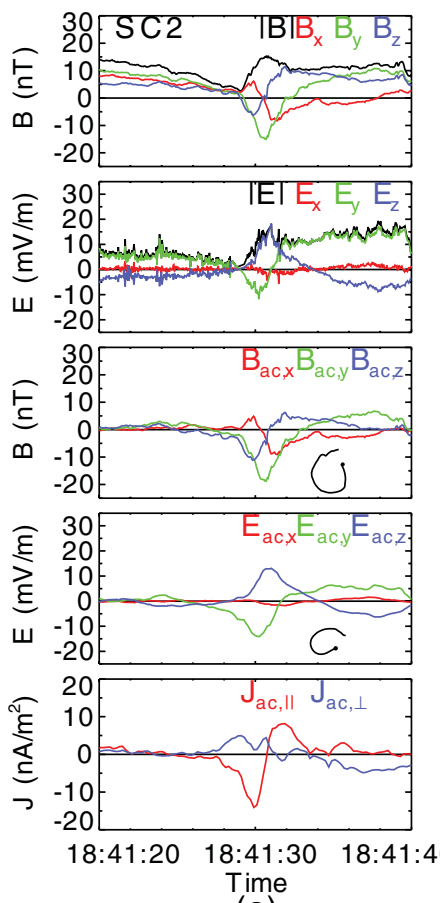

(a)
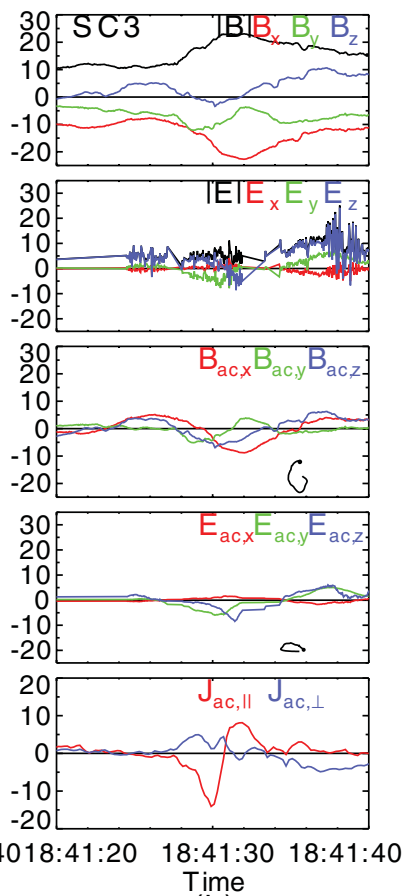

(b)
FIG. 2 (color). $\quad \mathbf{B}(22.5 \mathrm{~Hz}), \mathbf{E}(25 \mathrm{~Hz})$, and derived quantities. The top two rows show magnetic and electric field components and intensities. $E_{z}$ was computed assuming $\mathbf{E} \cdot \mathbf{B}=0$ [16], using measured $\mathbf{B}$ and spin-plane $E_{x}$ and $E_{y}$ components. The third and fourth rows show detrended $\delta \mathbf{B}$ and $\delta \mathbf{E}$ used to compute $\mathbf{k}$ and $V_{\mathrm{ph}}$. Inset hodograms are in the planes perpendicular to $\mathbf{k}$ (dots show the start). The bottom row shows $\langle J\rangle$ determined using four spacecraft data. 
frame $V_{\mathrm{ph}}^{\prime}=V_{\mathrm{ph}}-\mathbf{k} \cdot \mathbf{V}$ is $\sim 212 \pm 11.2 \mathrm{~km} \mathrm{~s}^{-1}$ (spinaveraged) and is comparable to $V_{A} \sim 200-250 \mathrm{~km} \mathrm{~s}^{-1}$ (also true for the pulse observed in the CS by SC3 at 1843 UT). The pulse detected by SC3 in the PSBL showed that $\delta \mathbf{B}$ and $\delta \mathbf{E}$ were less clearly defined. For this pulse, $\mathbf{k}=(0.525,0.724,-0.447)$, and the angle between $\mathbf{k}$ and B $\sim 54^{\circ}$ with a right-hand elliptical polarization. The plasma frame phase speed was $\sim 10.2 \pm 26.9 \mathrm{~km} \mathrm{~s}^{-1}$. Calculation of $\mathbf{J}=\nabla \times \mathbf{B} / \mu_{0}$ within the CS showed that $\langle J\rangle$ was small except during the pulses when $J_{\|}$attained $\sim 15 \mathrm{nA} / \mathrm{m}^{2}$. It reversed sign as $\mathbf{B}$ rotated so as to maintain an antisunward direction $\left(J_{x}<0\right)$. The current carriers are not the measured ions that were flowing in the opposite direction. Instead, they are likely to be the field-aligned electrons streaming in the sunward direction (see below).

Solitary pulses are seen often in the CS. However, most of the pulses are not as coherent as the example at 1841:30 UT. We attribute the coherence of the pulse detected by $\mathrm{SC} 2$ as resulting from a chance encounter with an event that is strongly localized in space (perhaps restricted to the CS) and in time. The source dimension was smaller than the SC separation, $\sim 1700 \mathrm{~km}$ (Larmor radius of 1$2 \mathrm{keV} p^{+}$for $B \sim 2 \mathrm{nT}$ ). However, the CS dynamics have global effects, and the pulses can be convected and be detected in the PSBL, for example. The coherent features dissipate in a short distance and time. Ten pulses were identified in the HPR on 12 August 2001; the five in the CS always had $V_{\perp}>V_{A}$, and five in the PSBL had $V_{\perp}<V_{A}$.

The possibility that the coherent pulse represents the passage of a magnetic island or flux rope past the spacecraft has been ruled out by the nonzero phase speed for the pulse in the plasma rest frame (such structures would convect with reconnection exhaust flows). In addition, traversals through a flux rope would be indicated by bipolar signatures in two components of $\mathbf{B}$ and a unipolar signature in the third that is oriented along its axis. Near symmetry of the bipolar $B_{x}$ and $B_{z}$ (Fig. 2) would suggest that the cluster had passed near the center of a flux rope, but the reversal of the sign in the unipolar component $B_{y}$ is hard to reconcile with present models; it would indicate a reversal in the direction of the guide field at the edges of the structure. An attempt to transform away the sign reversal by rotations about $X_{\mathrm{GSE}}$ and about the minimum variance direction (expected to be perpendicular to such an object's symmetry axis) found no coordinates that removed the sign reversal.

In the plasma sheet exterior to the HPR, the distributions were isotropic, but inside the region exhibited strong beams that accounted for the fast flows. Figure 3 shows ion data (12 s) from before, during, and after the pulse observed at 1841:35 UT. These are 2D slices through the 3D distributions with the top two rows showing slices of phase space in field-oriented coordinates $V_{\|} \times V_{\perp}$ and $V_{\perp 0} \times V_{\perp 1}$, and the bottom showing GSE coordinates $V_{x} \times V_{z}$. To guide the eye, magenta (orange) projections of the $B$ field $\left(X_{\mathrm{GSE}}\right)$ directions are drawn through the peaks

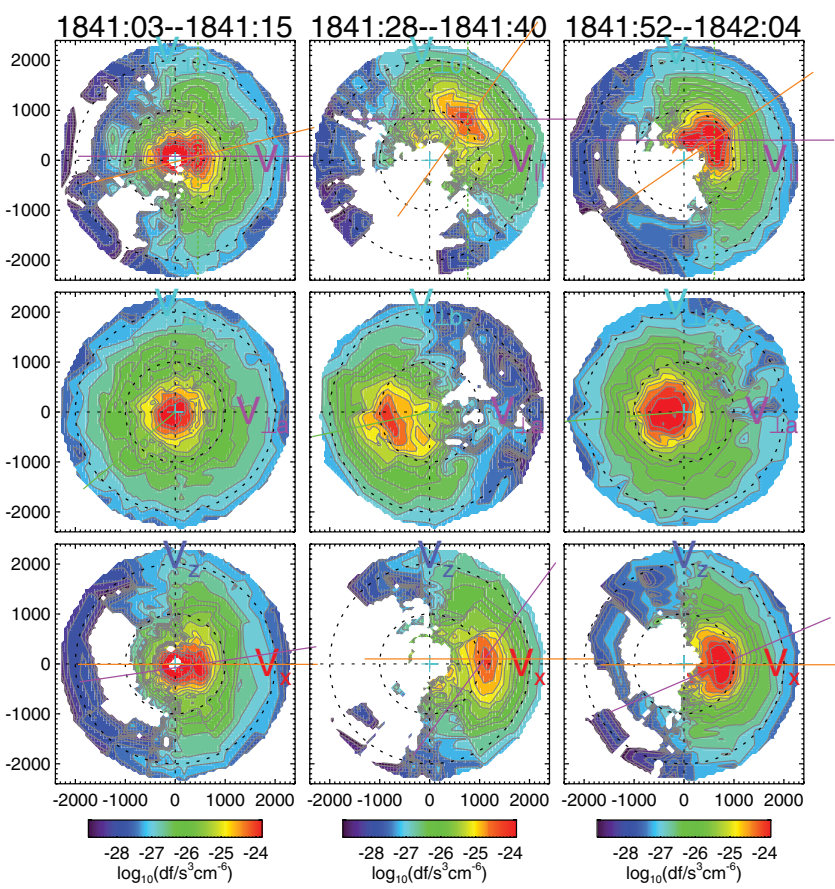

FIG. 3 (color). Phase space density of ions in the spacecraft frame detected on SC1 which was closest to SC2 (instrument on SC2 not operating). Ion distributions before (left), during (center), and after (right) the pulse are shown. $B$ field averaged over $12 \mathrm{~s}$ for the field-organized slices.

of the beams. In the $V_{\|} \times V_{\perp}$ slices, a lack of symmetry about the magenta lines shows that the ions are not well organized by $\mathbf{B}$, as is corroborated by displacements of the beams from the origin in the $V_{\perp 0} \times V_{\perp 1}$ cuts. The GSE plots exhibit near symmetry of the beams about $X_{\mathrm{GSE}}$, and the poor organization by $\mathbf{B}$ indicates that the beams are weakly magnetized in the HPR.

The electron distributions [17] from SC2 obtained within the plasma sheet were Maxwellian [Fig. 4(a)]. The distributions became anisotropic with $T_{\|}>T_{\perp}$ just prior to the spacecraft's entry into the HPR [Fig. 4(b)]. Within the solitary pulse, the distributions had a flat top shape with maximum phase space densities of $1 \times 10^{-29} \mathrm{~s}^{3} \mathrm{~cm}^{-6}$

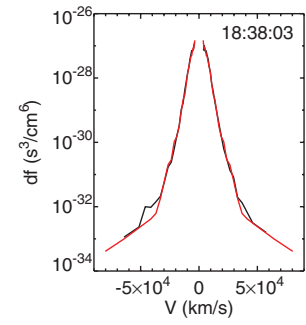

(a)

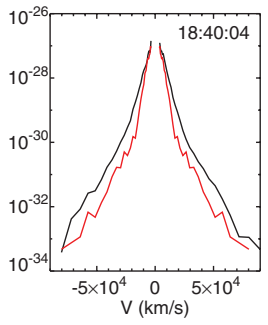

(b)

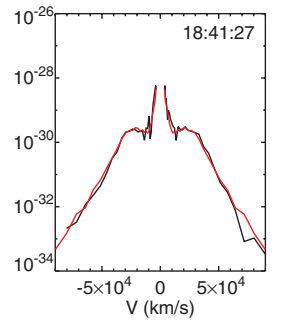

(c)
FIG. 4 (color). Parallel (black) and perpendicular (red) cuts of phase space densities for electrons from SC2. (a) Plasma sheet, (b) prior to HPR, and (c) within the solitary pulse. 


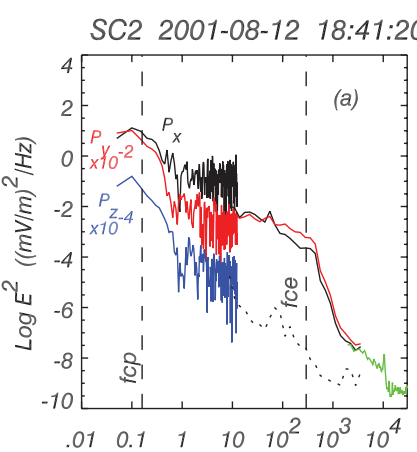
SC3 2001-08-12 18:41:20--18:41:40
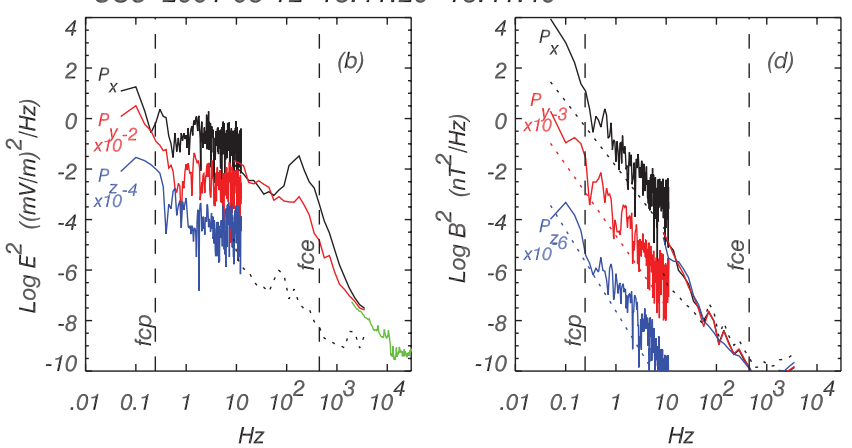

FIG. 5 (color). Power spectra of $\mathbf{E}$ (left) and $\mathbf{B}$ (right) fields from SC 2 and 3. The power of $E_{x}, E_{y}, E_{z}$ and $B_{x}, B_{y}, B_{z}$ for $f<12.5 \mathrm{~Hz}$ shown in black, red, and blue. For $f>8 \mathrm{~Hz}$, the power is the sum of the measurements in the spin plane, and $E_{x}$, $E_{y}$ and $B_{x}, B_{y}, B_{z}$ are plotted in the same colors. $\mathbf{E}$ measurements are extended to $\sim 30 \mathrm{kHz}$ (green). Dotted lines for $f>$ $8 \mathrm{~Hz}$ are instrument background noise.

[Fig. 4(c)]. Therefore, most of the low-energy $(<7 \mathrm{keV}$; $v<4.96 \times 10^{4} \mathrm{~km} \mathrm{~s}^{-1}$ ) electrons originally present were absent. These are similar in appearance to electron distributions seen in shock layers [18]. After the pulse, the distributions returned to a Maxwellian form.

$\mathbf{E}$ and $\mathbf{B}$ data from field and wave instruments $[15,19,20]$ were combined to obtain power spectra (Fig. 5). Wave power was enhanced within the fast flowing plasmas, and the strongest intensification occurred for $V_{\perp}>V_{A}$ flows. Enhanced electrostatic $(\delta \mathbf{E})$ fluctuations were observed to near the plasma $\left(f_{p e}\right)$ and upper hybrid frequencies $\left(f_{\mathrm{uh}} \sim 10 \mathrm{kHz}\right)$ and EM waves $(\delta \mathbf{B})$ up to the electron cyclotron frequency $\left(f_{c e}\right)$, consistent with the behavior of extreme low frequency and very low frequency whistler mode waves. The enhanced $\delta \mathbf{B}$ for $<1 \mathrm{~Hz}$ was associated with the proton cyclotron $\left(f_{\mathrm{cp}} \sim 0.17 \mathrm{~Hz}\right)$ and Alfvén waves. Enhanced E power between 1-10 Hz (near the local lower hybrid frequency) is due to the electrostatic spikes, which are purely electrostatic and common to the PSBL region.

The pulses in the CS are probably solitary structures of steepened ion cyclotron waves (left-hand polarized) and, in the PSBL, steepened Alfvén waves (right-hand polarized).
In a current carrying plasma with an electron thermal velocity that exceeds the phase velocity of Alfvén waves (for the present case, the thermal velocity of $2 \mathrm{keV}$ electrons is $v_{\mathrm{th}}^{e} \sim 2 \times 10^{4} \mathrm{~km} \mathrm{~s}^{-1} \gg V_{A} \sim 300 \mathrm{~km} \mathrm{~s}^{-1}$ ), transit time damping can couple energy from electrons to ions, and ion waves can grow. The association of $J_{\|}$is predicted for Alfvén wave solitary structures [21]. The waves involved could also be rotation waves [22] that can be excited when $\mathrm{O}^{+}$is present $\left(\sim 4 \% \mathrm{O}^{+}\right.$was present).

Our observations have shown that the geomagnetic tail can operate on Larmor time and size scales. The as-yet-tobe-identified mechanisms involve high- $\beta$ plasmas in which ions do not follow simply combined Larmor and drift motions, but the electrons, still tied to the $B$ field, play an important role. These features may be expected of diffusion regions in kinetic magnetic reconnection models [23], cross-tail current disruption models [24], and kinetic ballooning instabilities [25]. Plans are underway to combine data analysis and simulation models to test and validate plasma theories and models.

The research at UC Berkeley is funded by a cluster data analysis grant, NASA Grant No. NNG04GF23G.

*parks@ssl.berkeley.edu

[1] M. Shay et al., Geophys. Res. Lett. 30, 1145 (2003).

[2] V. Angelopoulos et al., J. Geophys. Res. 97, 4027 (1992).

[3] D. A. Gurnett, J. Geophys. Res. 79, 4227 (1974).

[4] G. K. Parks et al., Space Sci. Rev. 95, 237 (2001).

[5] B. Tsurutani and E. Smith, Geophys. Res. Lett. 13, 259 (1986).

[6] P. Louarn et al., Geophys. Res. Lett. 21, 1847 (1994).

[7] M. Temerin, M. Waldorf, and F. Mozer, Phys. Rev. Lett. 43, 1941 (1979).

[8] H. Matsumoto et al., Geophys. Res. Lett. 21, 2915 (1994).

[9] J. Pickett et al., Nonlin. Proc. Geophys. 12, 181 (2005).

[10] C. Cattell et al., Phys. Chem. Earth C 26, 97 (2001).

[11] K. Stasiewicz, Phys. Rev. Lett. 93, 125004 (2004).

[12] H. Rème et al., Ann. Geophys. 19, 1303 (2001).

[13] R. Nakamura et al., Geophys. Res. Lett. 29, 2140 (2002).

[14] A. T. Y. Lui et al., Geophys. Res. Lett. 32, L06101 (2005).

[15] A. Balogh et al., Ann. Geophys. 19, 1207 (2001).

[16] G. Gustafsson et al., Ann. Geophys. 19, 1219 (2001).

[17] A. Johnstone et al., Space Sci. Rev. 79, 351 (1997).

[18] W. C. Feldman et al., Phys. Rev. Lett. 49, 199 (1982).

[19] N. Cornilleau-Wehrlin et al., Ann. Geophys. 21, 437 (2003).

[20] P. Décréau et al., Ann. Geophys. 19, 1241 (2001).

[21] A. Hasegawa and C. Ubero, DOE/TIC Report No. 11197 (U.S. Department of Energy, Washington, DC, 1982).

[22] G. Ganguli and L. Rudakov, Phys. Plasmas 12, 042110 (2005).

[23] J. Drake et al., Geophys. Res. Lett. 33, L13 105 (2006).

[24] A. T. Y. Lui, Space Sci. Rev. 113, 127 (2004).

[25] C. Z. Cheng and A. T. Y. Lui, Geophys. Res. Lett. 25, 4091 (1998). 